\title{
Versatile fabrication and integration method of optical oxygen sensors in organ-on-chips
}

\author{
Elsbeth G.B.M. Bossink, Juliëtte V.M. Slob, Dorothee Wasserberg, Loes I. Segerink, and Mathieu Odijk \\ BIOS Lab on a chip group, MESA+ Institute, University of Twente, Enschede, the Netherlands \\ e.g.b.m.bossink@utwente.nl
}

\begin{abstract}
Oxygen concentration is an essential parameter in in vitro cell cultures as it affects cell viability, cellular activity, cell differentiation, and can be a measure for cellular response. Organ-on-chip $(\mathrm{OoC})$ devices provide an in vitro cell culture method, aiming to mimic the in vivo situation better than conventional cell culture methods. Oxygen control in these $\mathrm{OoC}$ devices is very meaningful especially for organs with inherently large oxygen concentration gradients, such as the gut. Here, we show a versatile fabrication method for optical oxygen sensor patches based on platinum tetrakis (pentafluorophenyl) porphyrin (PtTFPP) in polystyrene (PS). The PtTFPP/PS sensors show a linear Stern Volmer calibration plot. The proposed fabrication method allows abundant design freedom regarding both the sensor patches (size and shape) and integration (site and number of patches) in a typical two-channel $\mathrm{OoC}$ device.
\end{abstract}

Keywords-Optical oxygen sensor, Organ-on-chip, Platinum tetrakis (pentafluorophenyl) porphyrin, PtTFPP, Polystyrene

\section{INTRODUCTION}

The definition 'Organ-on-chips' (OoC) is used for in vitro microfluidic cell culture devices, which are often made of polydimethylsiloxane (PDMS), and considered promising alternatives for the conventional in vitro models or animal testing [1]-[3]. There is a lot of interest in oxygen control in $\mathrm{OoC}$ devices, as oxygen is a crucial molecule in the metabolism of human cells since both a deficiency as an abundance of oxygen can cause cell damage or even cell death [4], [5]. By measuring the (change of) oxygen concentration a wealth of information on cell viability, cellular activity, and cell responses can be provided, such as shown in [6].

The majority of the optical oxygen sensors are based on the quenching of the phosphorescence of metalloporphyrins by oxygen [4], [7], [8]. Platinum(II) porphyrins are known for their high quantum yields and long-live phosphorescence [9], beneficial for oxygen measurements. The sensing principle on which these kinds of sensors rely, is described in detail in [7]. Specifically, platinum tetrakis (pentafluorophenyl) porphyrin (PtTFPP) has a high photostability [7], [10] and is the most used oxygen sensitive dye [8].

Optical oxygen sensors based on metalloporphyrins for OoC devices are already shown in several different forms (described in more detail in [4], [11]), e.g. thin sensor films [12], [13], dye-coated optical fibers [14] or micro- and nanoparticles [15]. However, these sensor types all have their own disadvantages for measuring oxygen concentration during cell culture on specific sites; at or along the membrane in a two-channel OoC. For example, sensor films [12], [13], or sensor spots [16] are often integrated in an OoC device with only one cell culture channel. They are spin coated on or imprinted in a substrate, making it complicated to integrate them (at the membrane) in a two-channel device. Dye-coated optical fibers have to be physically inserted into the OoC, and this method can be tedious when multiplexing is desired. Oxygen sensors can also be applied in a separate sensor module in series of the OoC [17], which inherently makes it difficult to monitor the oxygen concentration at specific sites along the OoC. Oxygen sensing micro- and nanoparticles are impractical for

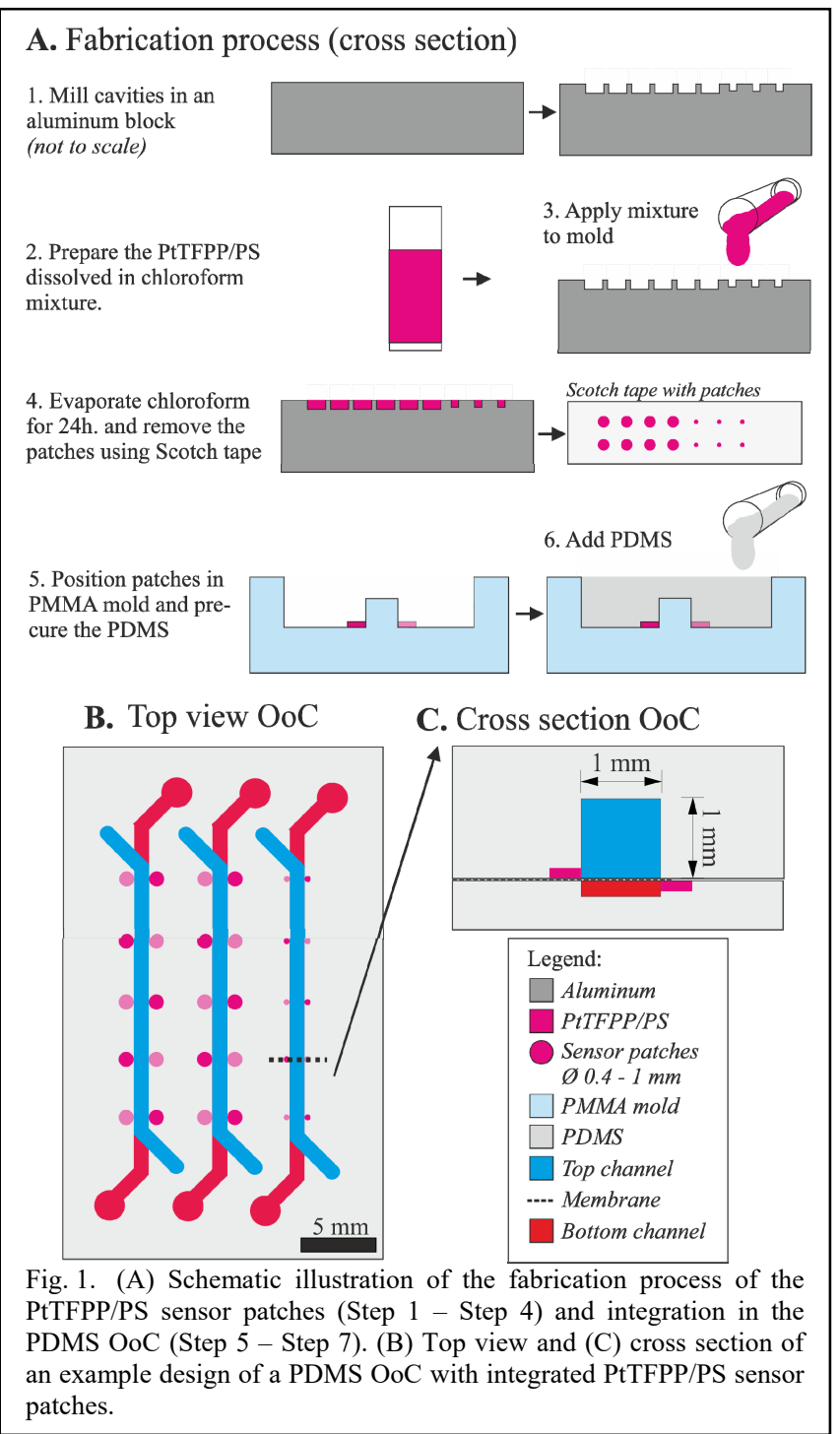


(long-term) continuous oxygen measurements during cell culture under flow in an OoC. Microparticles can accumulate in the culture channel and nanoparticles will be taken up by mammalian cells.

Here, we present a versatile method to fabricate optical oxygen sensor patches, based on PtTFPP in a polystyrene (PS) matrix which accommodates abundant design freedom regarding the sensor patches (size and shape). Furthermore, we show the integration in a typical PDMS OoC, where the (several) sensor patches can be placed in the PDMS OoC at any site of interest.

\section{EXPERIMENTAL}

\section{A. PtTFPP/PS patch fabrication}

PS (Mw 280,000, Sigma-Aldrich) is dissolved in chloroform (Sigma-Aldrich) $(7 \% \mathrm{w} / \mathrm{v})$, where after this mixture is added to PtTFPP (1\%w/v) (Frontier Scientific) and vortexed thoroughly. The PtTFPP/PS in chloroform mixture is pipetted on an in-house milled aluminum mold with $0.4 \mathrm{~mm}$ to $1 \mathrm{~mm}$ diameter cavities with a depth of approximately $200 \mu \mathrm{m}$ and $500 \mu \mathrm{m}$. The chloroform is left to evaporate for 24 hours. Using Scotch tape, the PtTFPP/PS patches can be removed from the aluminum mold (see Fig. 1(A) step 1-4).

\section{B. Integration of patches in a PDMS OoC}

PDMS (Sylgard 184 Sililcone elastomer kit, Dow Corning) was mixed $(10: 1 \% \mathrm{w} / \mathrm{w})$ and degassed for approximately 2 hours. The PtTFPP/PS patches were 'glued' into an in-house micromilled poly(methyl methacrylate) (PMMA) mold, using uncured PDMS (10:1\%w/w), which is then pre-cured for 30 minutes at $60^{\circ} \mathrm{C}$. Subsequently, the PMMA mold was completely filled with PDMS (10:1 $\% \mathrm{w} / \mathrm{w}$ ) and cured for 4 hours at $60^{\circ} \mathrm{C}$ (see Fig. 1(A) step 5). This process can be performed for both PDMS top and bottom layer, followed by assembly of the OoC (see Fig. 1(B) and (C)). Alternatively, a PDMS layer could also be bonded to a glass slide using oxygen plasma, thereby creating a one-channel device.

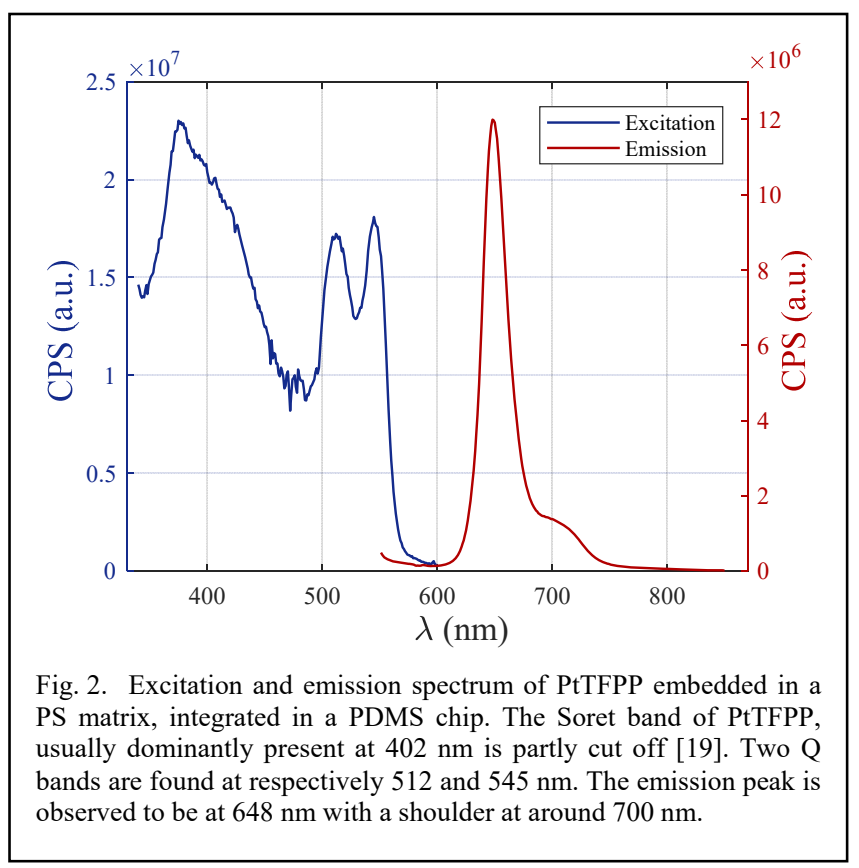

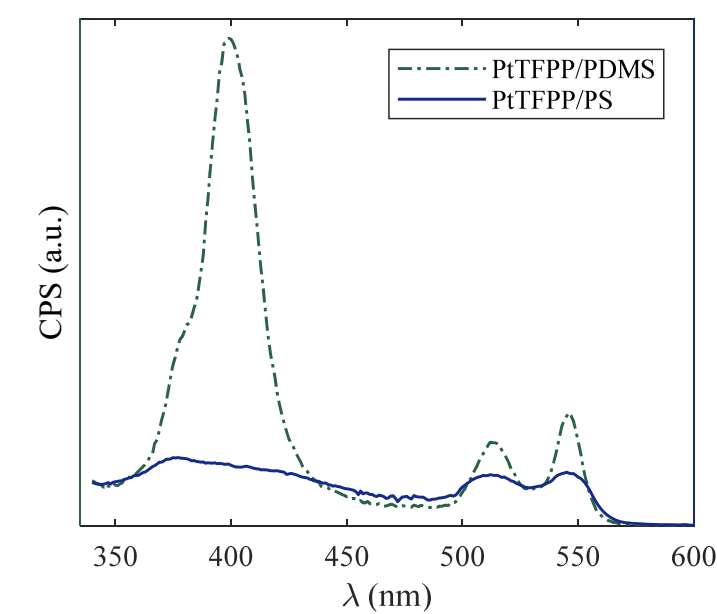

Fig. 3. The two excitation spectra of PtTFPP embedded in a PDMS matrix (PtTFPP/PDMS), and PtTFPP embedded in PS and integrated in a PDMS chip (PtTFPP/PS). The excitation spectrum of PtTFPP/PDMS is similar as seen in literature [19]. The excitation spectrum of PtTFPP/PS differs from reported before [10].

\section{Excitation and emission spectra}

The excitation and emission spectra of PtTFPP/PS were measured using a FluoroMax-4 photo spectrometer with lifetime extension (Horiba Scientific). The excitation spectrum was measured with emission at $650 \mathrm{~nm}$ and emission spectrum was measured with excitation at $540 \mathrm{~nm}$.

To study the effect of the polymer matrix in which the PtTFPP is embedded, we studied the excitation spectra of both PtTFPP in a PDMS matrix (PtTFPP/PDMS) and PtTFPP in a PS matrix, integrated in a PDMS chip (PtTFPP/PS in PDMS). The PtTFPP/PDMS was obtained by dissolving PDMS in toluene (Sigma Aldrich) adding $1 \% \mathrm{w} / \mathrm{v}$ PtTFPP, and subsequent curing at $60^{\circ} \mathrm{C}$.

\section{Calibration of PtTFPP/PS sensor}

PtTFPP/PS sensors were calibrated for gaseous oxygen ranging from instrumental air $(210 \mathrm{hPa}$ partial oxygen pressure) to nitrogen ( $0 \mathrm{hPa}$ partial oxygen pressure). A microscope with color CCD camera (FLIR Grasshopper 3, U232S6C) and a Chroma 49012-ET-FITC/EGFP long pass filter was used to record images. The images were processed using the software ImageJ (version 1.52a).

\section{RESULTS AND DISCUSSION}

\section{A. Excitation and emission spectra}

The excitation and emission spectra of the PtTFPP/PS in PDMS are shown in Fig. 2. The usually intense Soret band at $402 \mathrm{~nm}$ seen in PtTFPP is partly cut off, compared to results shown in literature [18], [19]. The two Q bands are present around 512 and $545 \mathrm{~nm}$. The emission spectrum is comparable as shown before as a peak is observed at $648 \mathrm{~nm}$ with a shoulder at around $700 \mathrm{~nm}$; this is slightly shifted with regard to values found in literature [20].

To clarify the difference of our excitation spectrum of PtTFPP/PS in a PDMS chip compared to literature [10], we compare in Fig. 3 PtTFPP/PS in PDMS (solid line) with PtTFPP/PDMS (dashed line). The excitation spectrum of PtTFPP/PDMS resemble absorption spectra as observed in 


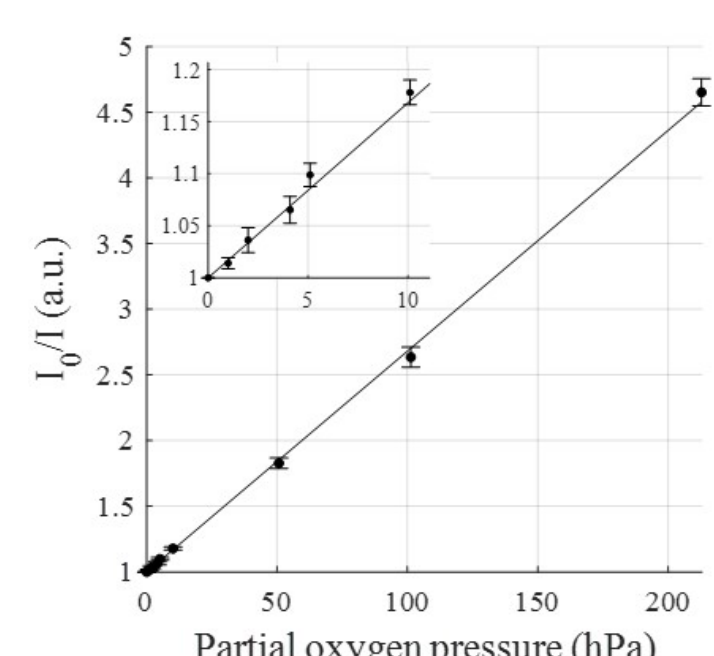

Fig. 4. The linear Stern-Volmer calibration plot of PtTFPP/PS sensors (not in PDMS) for gaseous oxygen. $\mathrm{I}_{0}$ is the intensity under pure nitrogen $(0 \%$ oxygen $), I$ is the measured intensity at a certain oxygen concentration. A Stern-Volmer coefficient $\left(\mathrm{K}_{\mathrm{sv}}\right)$ of 0.0168 $\mathrm{hPa}^{-1}$ and a $\mathrm{R}^{2}=0.998$ was observed. Averages and +/- standard deviation are shown $(n=3)$.

literature [19], [20], which is also shown for PtTFPP/PS [10], [18]. So, the integration of PtTFPP/PS sensors in PDMS specifically causes an effect on the excitation spectrum.

\section{B. Calibration of PtTFPP/PS sensor}

When calibrating the PtTFPP/PS sensors for gaseous oxygen, a linear Stern-Volmer relationship was obtained (Fig. 4), with a Stern-Volmer coefficient $\left(\mathrm{K}_{\mathrm{sv}}\right)$ of 0.0168 $\mathrm{hPa}^{-1}$ and a $\mathrm{R}^{2}=0.998$. Such linear Stern-Volmer plot is earlier shown in literature [10].

We are aware that intensity based sensing is prone to measuring errors, since it is affected by various factors such as: the stability of the light source, background luminescence, concentration and distribution of the dye in probes or patches, photobleaching, and scattering [7], [8]. Lifetime measurements as presented in [18] for PtTFPP/PS sensors, can overcome the mentioned disadvantages of intensity based oxygen measuring [4]. For lifetime measurements only a modulated excitation source is required, and a detector which can sample within the lifetime of the PtTFPP. Future experiments will focus on characterizing the oxygen sensor patches in an organ-onchip during cell culture employing lifetime measurements.

\section{Fabricated device}

Fig. 5 shows two example PDMS chips with integrated PtTFPP/PS sensor patches (1 mm diameter) to illustrate the flexibility of the integration method. Fig. 5A shows a PDMS top channel containing three channels and 15 integrated sensor patches. Fig. 5B shows a PDMS OoC containing two parallel channels with sensor patches in both top and bottom PDMS layer, of which a cross section is shown in Fig. 5C.

The sensor patches can be placed anywhere in the PDMS chip as desired by gluing or pouring PDMS in the mold up to a certain height, partly curing, and subsequently adding the sensor patches. We fabricated patches ranging from $0.4-1 \mathrm{~mm}$ in diameter. The only limitation of the size and shape of the sensor patches is the minimal diameter mill available for milling the cavities in the aluminum mold.

The sensor patches can be used to study or confirm the effect of oxygen modulation within an OoC such as shown in [5], [13], [21]. This oxygen modulation can be important for cell differentiation on-chip [4] or e.g. co-culture with microbiota [22]. Furthermore, the sensor patches can be used to measure oxygen concentration during cell culture on-chip, providing valuable information about e.g. cell viability, activity and cellular response [4], [5].

\section{CONCLUSION}

In conclusion, we showed a versatile fabrication method for optical oxygen sensor patches based on PtTFPP in a PS matrix. The only limitation regarding the size and shape of the sensor patches is the minimal diameter mill available. The PtTFPP/PS sensors show a linear Stern-Volmer plot. We also showed the integration of the sensor patches in both channels of a two-channel PDMS OoC. The integration method is flexible, allows multiplexing and integration of the sensors in the PDMS OoC at any desired site.

\section{ACKNOWLEDGMENTS}

Funding source: This project has been funded by a Building Blocks of Life grant from the Netherlands Organization for Scientific Research (NWO), grant no. 737.016.003.

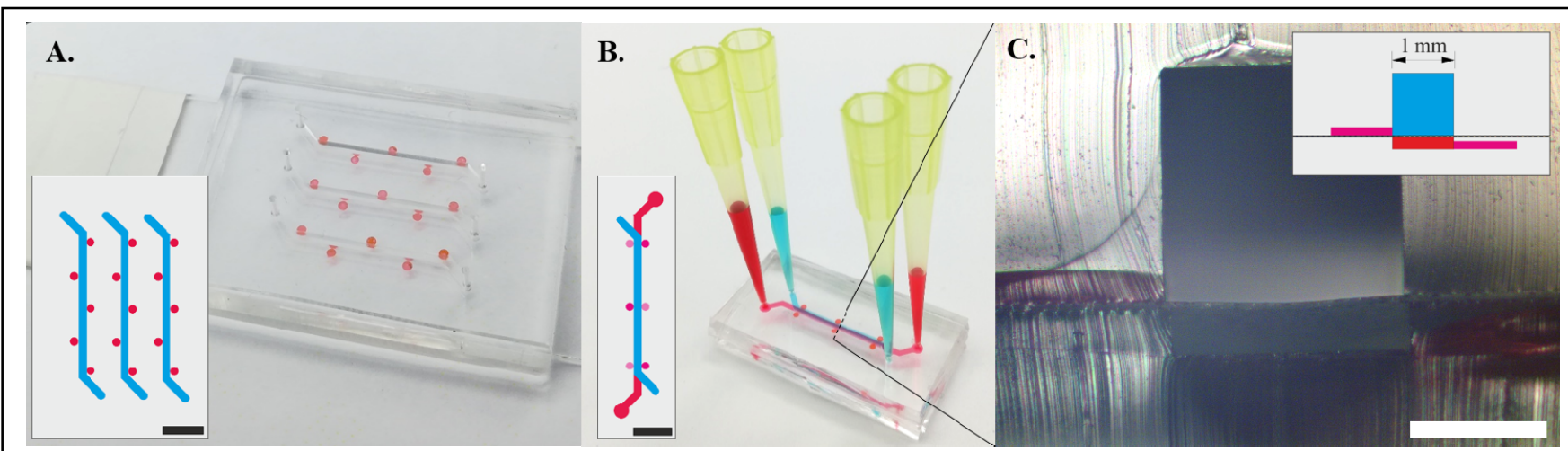

Fig. 5. Examples of fabricated PDMS chips with integrated PtTFPP/PS patches. (A) A fabricated PDMS top channel with five red, circular PtTFPP/PS patches (1 mm diameter) in each channel. The PDMS layer is bonded to a glass slide. Schematic illustration of a top view is shown in the left bottom corner with the scale bar representing $5 \mathrm{~mm}$. (B) A typical PDMS OoC containing two parallel channels, with integrated sensor patches (1 mm diameter), three per channel. Schematic illustration of a top view is shown in the left bottom corner with the scale bar representing 5 mm. (C) Brightfield microscopy image of a cross section of an OoC as shown in Fig. 5b. Two sensor patches are shown next to the top and bottom channels according to the schematic illustration in the right upper corner. Scale bar represents $500 \mu \mathrm{m}$. 


\section{REFERENCES}

[1] S. N. Bhatia and D. E. Ingber, "Microfluidic organs-on-chips," Nat. Biotechnol., vol. 32, no. 8, pp. 760-772, 2014, doi: 10.1038/nbt.2989.

[2] D. E. Ingber, "Reverse Engineering Human Pathophysiology with Organs-on-Chips," Cell, vol. 164, no. 6, pp. 1105-1109, 2016, doi: 10.1016/j.cell.2016.02.049.

[3] S. Reardon, “'Organs-on-chips' go mainstream,” Nature, vol. 523, no. 7560, p. 266, 2015, doi: 10.1038/523266a.

[4] P. E. Oomen, M. D. Skolimowski, and E. Verpoorte, "Implementing oxygen control in chip-based cell and tissue culture systems," Lab Chip, vol. 16, pp. 3394-3414, 2016, doi: 10.1039/c6lc00772d.

[5] M. D. Brennan, M. L. Rexius-Hall, L. J. Elgass, and D. T. Eddington, "Oxygen control with microfluidics," Lab Chip, vol. 14, no. 22, pp. 4305-4318, 2014, doi: 10.1039/c4lc00853g.

[6] J. Marzioch et al., "On-chip photodynamic therapy-monitoring cell metabolism using electrochemical microsensors," Lab Chip, vol. 18, no. 22, pp. 3353-3360, 2018, doi: 10.1039/C8LC00799C.

[7] M. Quaranta, S. M. Borisov, and I. Klimant, "Indicators for optical oxygen sensors," Bioanal. Rev., vol. 4, no. 2-4, pp. 115-157, 2012, doi: 10.1007/s12566-012-0032-y.

[8] X. D. Wang and O. S. Wolfbeis, "Optical methods for sensing and imaging oxygen: Materials, spectroscopies and applications," Chem. Soc. Rev., vol. 43, no. 10, pp. 3666-3761, 2014, doi: $10.1039 / \mathrm{c} 4 \mathrm{cs} 00039 \mathrm{k}$.

[9] J. R. Sommer, A. H. Shelton, A. Parthasarathy, I. Ghiviriga, J. R. Reynolds, and K. S. Schanze, "Photophysical properties of nearinfrared phosphorescent $\pi$-extended platinum porphyrins," Chem. Mater., vol. 23, no. 24, pp. 5296-5304, 2011, doi: $10.1021 / \mathrm{cm} 202241 \mathrm{e}$.

[10] S. K. Lee and I. Okura, "Photostable Optical Oxygen Sensing Material: Platinum Tetrakis(pentafluorophenyl)porphyrin Immobilized in Polystyrene," Anal. Commun., vol. 34, no. 6, pp. 185188, 1997, doi: 10.1039/a701130j.

[11] S. M. Grist, L. Chrostowski, and K. C. Cheung, "Optical oxygen sensors for applications in microfluidic cell culture," Sensors (Switzerland), vol. 10, no. 10, pp. 9286-9316, 2010, doi: 10.3390/s101009286.

[12] H. Zirath et al., "Every breath you take: Non-invasive real-time oxygen biosensing in two- and three-dimensional microfluidic cell models," Front. Physiol., vol. 9, no. JUL, pp. 1-12, 2018, doi: 10.3389/fphys.2018.00815.
[13] P. C. Thomas, S. R. Raghavan, and S. P. Forry, "Regulating oxygen levels in a microfluidic device," Anal. Chem., vol. 83, no. 22, pp. 8821-8824, 2011, doi: 10.1021/ac202300g.

[14] E. J. Park, K. R. Reid, W. Tang, R. T. Kennedy, and R. Kopelman, "Ratiometric fiber optic sensors for the detection of inter- and intracellular dissolved oxygen," J. Mater. Chem., vol. 15, no. 27-28, pp. 2913-2919, 2005, doi: 10.1039/b502981c.

[15] C. O’Donovan, J. Hynes, D. Yashunski, and D. B. Papkovsky, "Phosphorescent oxygen-sensitive materials for biological applications," J. Mater. Chem., vol. 15, no. 27-28, pp. 2946-2951, 2005, doi: 10.1039/b501748c.

[16] J. Ehgartner et al., "Online analysis of oxygen inside silicon-glass microreactors with integrated optical sensors," Sensors Actuators, B Chem., vol. 228, pp. 748-757, 2016, doi: 10.1016/j.snb.2016.01.050.

[17] Y. S. Zhang et al., "Multisensor-integrated organs-on-chips platform for automated and continual in situ monitoring of organoid behaviors," Proc. Natl. Acad. Sci. U. S. A., vol. 114, no. 12, pp. E2293-E2302, 2017, doi: 10.1073/pnas.1612906114.

[18] Y. Amao, K. Asai, and I. Okura, "Oxygen sensing based on lifetime of photoexcited triplet state of platinum porphyrin-polystyrene film using time-resolved spectroscopy," J. Porphyr. Phthalocyanines, vol. 4, no. 3, pp. 292-299, 2000, doi: 10.1002/(SICI)10991409(200004/05)4:3<292::AID-JPP216>3.0.CO;2-W.

[19] S. W. Lai et al., "Electronic spectroscopy, photophysical properties, and emission quenching studies of an oxidatively robust perfluorinated platinum porphyrin," Inorg. Chem., vol. 43, no. 12, pp. 3724-3732, 2004, doi: 10.1021/ic049902h.

[20] C. Arunkumar, F. R. Kooriyaden, X. Zhang, S. Sujatha, and J. Zhao, "Fluorinated: Meso -tetraaryl Pt(II)-porphyrins: Structure, photophysical, electrochemical, and phosphorescent oxygen sensing studies," New J. Chem., vol. 41, no. 12, pp. 4908-4917, 2017, doi: 10.1039/c7nj01141e.

[21] D. I. Walsh et al., "Emulation of Colonic Oxygen Gradients in a Microdevice," SLAS Technol., vol. 23, no. 2, pp. 164-171, 2018, doi: $10.1177 / 2472630317743425$.

[22] S. Jalili-Firoozinezhad et al., "A complex human gut microbiome cultured in an anaerobic intestine-on-a-chip," Nat. Biomed. Eng., vol. 3, no. 7, pp. 520-531, 2019, doi: 10.1038/s41551-019-0397-0. 